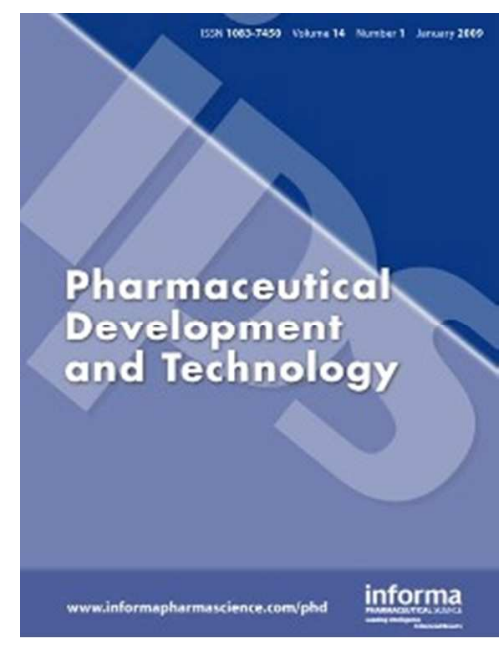

\title{
Penetration enhancer-containing vesicles (PEVs) as carriers for cutaneous delivery of minoxidil: in vitro evaluation of drug permeation by infrared spectroscopy
}

\begin{tabular}{|r|l|}
\hline Journal: & Pharmaceutical Development and Technology \\
\hline Manuscript ID: & LPDT-2012-0074.R1 \\
\hline Manuscript Type: & Original Research \\
\hline Date Submitted by the Author: & O3-Apr-2012 \\
\hline Complete List of Authors: & $\begin{array}{l}\text { Mura, Simona; Université Paris Sud, ; Università di Cagliari, } \\
\text { Manconi, Maria; Università di Cagliari, } \\
\text { Fadda, Anna; Università di Cagliari, } \\
\text { Sala, Maria; Università di Milano, } \\
\text { Perricci, Jacopo; Università di Milano, } \\
\text { Pini, Elena; Università di Milano, } \\
\text { Sinico, Chiara; Università degli Studi di Cagliari, Farmaco Chimico } \\
\text { Tecnologico }\end{array}$ \\
\hline Keywords: & \begin{tabular}{l} 
vesicles, FTIR imaging, stratum corneum \\
\hline
\end{tabular} \\
\hline
\end{tabular}

\section{SCHOLARONE"}

Manuscripts 


\section{Penetration enhancer-containing vesicles (PEVs) as carriers for cutaneous delivery of minoxidil: in vitro evaluation of drug permeation by infrared spectroscopy \\ Simona Mura ${ }^{\mathrm{a}, \mathrm{b}}$, Maria Manconi ${ }^{\mathrm{a}}$, Anna Maria Fadda ${ }^{\mathrm{a}}$, Maria Chiara Sala ${ }^{\mathrm{c}}$, Jacopo Perricci ${ }^{\mathrm{c}}$, Elena Pini $^{\mathrm{c}}$, Chiara Sinico* ${ }^{\mathrm{a}}$}

${ }^{a}$ Dipartimento Farmaco Chimico Tecnologico, Università degli Studi di Cagliari, Via Ospedale 72, 09124 Cagliari, Italy

${ }^{\text {b }}$ UMR CNRS 8612, IFR 141, Faculté de Pharmacie,Université Paris Sud, F- 92296

Châtenay-Malabry, France

c DISMAB-Sezione di Chimica Organica"A.Marchesini”,Università degli Studi di Milano, Via Venezian 21, 20133 Milano, Italy

\footnotetext{
Phone: +390706758555

Fax: +390706758553

e-mail: sinico@unica.it
}

* Corresponding author: Chiara Sinico

Dipartimento Farmaco Chimico Tecnologico,

Università degli Studi di Cagliari, Via Ospedale 72, 09124 Cagliari - Italy

Keywords: PEV, minoxidil, ATR-FTIR, FTIR imaging, stratum corneum, 


\begin{abstract}
Recently, we carried out a research on new liposomal systems prepared by using in their composition a few penetration enhancers which differ for chemical structure and physicochemical properties. The Penetration Enhancer-containing Vesicles (PEVs) were prepared by using soy lecithin and different amounts of three penetration enhancers, 2-(2ethoxyethoxy) ethanol (Transcutol $\left.{ }^{\circledR}\right)$, capryl-caproyl macrogol 8-glyceride (Labrasol®), and cineole .To study the influence of the PEVs on (trans)dermal delivery of minoxidil, in vitro diffusion experiments were performed through new born pig skin and the results were compared with that obtained applying the vesicular system without enhancer (control) after pretreatment of the skin with the various enhancers.

In this study Fourier Transform Infrared Spectroscopy (FTIR), Attenuated Total Reflectance FTIR (ATR-FTIR) and FTIR imaging were used to evaluate the effective penetration of minoxidil in the skin layers and to discover the influence of the enhancer on the drug topical delivery. These analytical studies allowed us to characterize the drug formulations and to evaluate the vesicle distribution into the skin. Recorded spectra confirmed that the vesicle formulations with penetration enhancers promoted drug deposition into the skin.
\end{abstract}




\section{Introduction}

The skin represents one of the most attractive sites for non-invasive administration of therapeutic agents, but the main obstacle to dermal and transdermal drug delivery is the barrier formed by the stratum corneum (SC) due to its brick and mortar-like structure that provides resistance to drug penetration. In order to obtain an enhanced drug delivery through the skin, topical formulations able to produce a temporary and reversible reduction of the $\mathrm{SC}$ resistance to diffusion of molecules are required.

Minoxidil (MX) is the most widely used drug for the topical treatment of androgenetic alopecia, an androgen dependent process, that is the mainly cause of hair loss in men and women with a genetic predisposition. The mechanism by which MX, topically applied, stimulates hair growth is not clearly understood and several different hypothesis have been reported from the 1980s onwards. Recently, has been reported that hair follicle dermal papilla cells represent the main target site for MX, which has a direct effect on the proliferation and apoptosis of these cells. The MX maximum effect is evident after 5 months of therapy, but it is not permanent. The treatment suspension causes an acceleration of hair loss 4-6 months. It is possible to find MX formulations containing ethanol, propylene glycol and water $[1,2]$. It is recommended twice a day application, but the chronic use is responsible of adverse effect, such as irritation, burning, itching [3], that reduce the compliance of users. To reduce side effects, and improve the therapeutic efficiency, there is the need to develop novel formulations for topical application. To increase MX accumulation in the skin layers in recent years different vesicular systems have been investigated. Lipid vesicles are reported to enhance topical drug delivery, with a reduction of the dose applied [4-12].

Recent approaches in modulating drug delivery through the skin led to the development of novel vesicular carriers with elastic properties called Transfersomes ${ }^{\circledR}$. These vesicles consist of phospholipids and an edge activator, that destabilizes the lipid bilayer and increases its deformability. The edge activator is a single chain surfactant molecule, which could be replaced with chemical penetration enhancers $[10,13]$. 
In the attempt to find new vesicular formulations able to optimize MX cutaneous delivery, in a previous work we prepared and characterized new liposomal systems prepared by using in their composition a few penetration enhancers (PEs) which differ for chemical structure and physicochemical properties [14]. The ability of the different penetration enhancers to produce elastic vesicles with soy lecithin and the influence of the Penetration Enhancer-containing Vesicles (PEVs) on (trans)dermal delivery of minoxidil were evaluated [15].

In this work so-called PEVs were prepared by using soy lecithin and three penetration enhancers, 2-(2-ethoxyethoxy)ethanol (Transcutol®), capryl-caproyl macrogol 8-glyceride (Labrasol ${ }^{\circledR}$ ), and cineole and Fourier Transform Infrared Spectroscopy (FTIR), Attenuated Total Reflectance FTIR (ATR-FTIR) and FTIR imaging were used to evaluate the effective penetration of minoxidil in the skin layer and to discover the influence of the enhancer on this topical delivery.

Over the last decade, ATR-FTIR technique has been used to characterize the structure and the properties of the stratum corneum and for the detection of drugs penetration after application on skin $[16,17]$. ATR-FTIR spectroscopy is also instructive with respect to the structural and conformational status of stratum corneum components and their modification after application of enhancers [18]. This technique has been extensively used to rapidly and noninvasively quantify in vivo the uptake of a chemical into the outermost, and least permeable, layer of human skin (the stratum corneum) [19-23]. Finally, it has also been used to understand the modifications of the lipid organization within the stratum corneum after application of different vehicles and to correlate this modification with the percutaneous penetration data $[24,25]$.

In this study Fourier transform infrared spectroscopy (FTIR), ATR-FTIR and FTIR imaging were used to evaluate the effective penetration of MX in the skin layer and to discover the influence of the enhancer on this topical delivery. The aim of this work was to investigate any change in the IR spectra of the skin after (i) pretreatment with penetration enhancers and subsequent application of 
MX liposomal dispersion and (ii) treatment with MX PEVs, to evaluate the effective penetration of the drug in the skin layers.

\section{Materials and methods}

Materials

Soy lecithin (SL) was obtained from Galeno (Potenza, Italy). Minoxidil (MX) (MW 209.25, 99\% purity) was purchased from Farma Labor (Bari, Italy). Dicetylphosphate (DCP), cineole (Cin), were purchased from Aldrich (Milan, Italy). 2-(2-ethoxyethoxy)ethanol (Transcutol ${ }^{\circledR}$, Tcr) and caprylcaproyl macrogol 8-glyceride (Labrasol ${ }^{\circledR}$ Lb) were a gift from Gattefossè, (Saint Priest, France). All the products and solvents were of analytical grade.

\section{HPLC method}

MX detection was performed at $231 \mathrm{~nm}$ using a high performance liquid chromatograph (HPLC) Alliance 2690 (Waters), equipped with a photodiode array detector and a computer integrating apparatus (Millennium 32). The column was a Nova-Pack C18 (60 ̊́, $4 \mu \mathrm{m})$ HPLC cartridge (Waters). The mobile phase was a mixture of methanol and water (80:20, $\mathrm{v} / \mathrm{v})$. The sample volume injected was $50 \mu \mathrm{l}$. The flow rate was $2.2 \mathrm{ml} / \mathrm{min}$. Retention time of MX was $1.4 \mathrm{~min}$. The detection limit was $10 \mathrm{ng} / \mathrm{ml}$. For concentrations in the range $0-50$ $\mu \mathrm{g} / \mathrm{ml}$ a good linearity was obtained $(\mathrm{R} 2=0.998 ; \mathrm{P}<0.001)$.

\section{Vesicle preparation and characterization}

Dehydrated-rehydrated vesicles (DRV) were prepared according to previously described methods [15].SL (60mg/ml), DCP (5.4 mg/ml), one PE $(20 \mathrm{mg} / \mathrm{ml})$ and MX (50mg/ml) were dissolved in chloroform. Then, lipid-drug mixture was deposited as a thin film in a round-bottom flask by rotoevaporating (Rotavapor Büchi R110, Switzerland) the chloroform under vacuum, which was 
applied for one hour to ensure total removal of solvent trace. The film was hydrated with distilled water under mechanical stirring at room temperature. Vesicle suspension was sonicated in a Soniprep 150 (MSE Crowley apparatus, Beckenham, UK) in 25 cycles of 10 seconds "on" and 10 seconds “off”. Liposomal dispersions, were freezed at $-20^{\circ} \mathrm{C}$ for 12 hours and then freeze-dried overnight using a Criotecnica freeze-drier apparatus (MM Cota Company, Roma, Italy) with 760 $\mathrm{mmHg}$ operative pressure at $80^{\circ} \mathrm{C}$. Vesicle were stored at $25^{\circ} \mathrm{C}$ for 30 days and successively vesicles were reconstituted by controlled rehydration in distilled water and mechanically shaken by a vortex (Velp scientifica, Italy) for 10 minutes. Each vesicle suspension was purified from nonincorporated drug by exhaustive dialysis for $4 \mathrm{~h}$ in distilled water at $5^{\circ} \mathrm{C}$ using Spectra-Por ${ }^{\circledR}$ membranes (12-14 kDa MW cut-off, 3nm pore size; Spectrum Laboratorie Inc., USA). Incorporation efficiency (E\%), expressed as the percentage of the drug amount initially used, was determined by high performance liquid chromatography (HPLC) after disruption of purified vesicles with a $0.025 \%$ Triton X-100 solution. Minoxidil content was quantified at $231 \mathrm{~nm}$ using a chromatograph Alliance 2690 (Waters, Italy), equipped with a photodiode array detector. The column was a Novo-Pack C18 (Waters, Italy) and the mobile phase was a mixture of methanol and water $(80: 20 \mathrm{v} / \mathrm{v})$ delivered at a flow rate of $2.2 \mathrm{ml} / \mathrm{min}$. The MX retention time was $1.4 \mathrm{~min}$.

In vitro skin penetration studies and skin pre-treatment.

Before use, the lyophilised MX loading vesicles with and without enhancer (control) were rehydrated with distilled water and mechanically shaken until a milky suspension was obtained. In vitro skin penetration and permeation studies were performed using vertical diffusion Franz cells with an effective diffusion area of $0.785 \mathrm{~cm}^{2}$. New born pig skin specimens were sandwiched securely between donor and receptor compartments, with the epidermis side facing the donor compartment. One-day-old Goland-Pietrain hybrid pigs $(1.2 \mathrm{~kg})$ were obtained from a local slaughterhouse. The receptor compartment was filled with $5.5 \mathrm{ml}$ of hydroalcoholic solution 
(water/ethanol 1/1) which was continuously stirred with a small magnetic bar and thermostated at $37^{\circ} \pm 1{ }^{\circ} \mathrm{C}$ throughout the experiments. $100 \mu$ of either MX entrapped vesicle with penetration enhancers or liposomal formulation without enhancer (control group) were placed onto the skin surface The receiving solution was withdrawn at regular intervals of 2 hours up to 8 hours, and replaced with an equivalent volume of hydro alcoholic solution to ensure sink conditions.

After 8 hours, the skin surface of specimens was washed 10 times with $1 \mathrm{ml}$ of distilled water then dried with filter paper. The stratum corneum (SC) was removed by striping with an adhesive tape Tesa ${ }^{\circledR}$ AG (Hamburg Germany). A piece of the adhesive tape was firmly pressed on the skin surface and rapidly pulled off with one fluent stroke. Ten stripping procedures were consecutively performed. The epidermis was separated from the dermis with a surgical sterile scalpel. The 10 tape strips, epidermis and dermis, were placed each in a vial with $3 \mathrm{ml}$ of methanol and then sonicated for four minutes in order to extract the drug. The tape and tissue suspension were centrifuged for 10 minutes at $10000 \mathrm{rpm}$, then the supernatants were filtered and assayed for their MX content by HPLC as described previously.

During pre-treatment studies, $100 \mu 1$ of solution of each penetration enhancer (at the same amount used in the PEVs formulations) was placed in the donor compartment of the diffusion cell. After incubation, skin surface was washed with distilled water and MX loading liposomes were placed on the skin samples. Then, the in vitro study was carried out for 8 hours as described above.

\section{Isolation of SC and viable epidermis.}

After the in vitro permeation and penetration studies epidermis was separated from the dermis by immersion of full thickness skin in a $60^{\circ} \mathrm{C}$ water bath for 2 minutes. It led possible to peel cycles of epidermis from the underlying tissue

The resulting sheets were rinsed with ultrapure water, dried at room temperature and stored in open boxes until used. 


\section{Preparation of samples}

Before physical analysis, lyophilized MXloading vesicles were re-hydrated with distilled water and mechanically shaken until an homogeneous dispersion was obtained.

For the spectroscopy study the epidermis specimens were compressed applying a strength of 3-4 ton using an hydraulic press to obtain a thinner and homogeneous sample.

\section{Fourier transform infrared spectroscopy (FT-IR).}

Solid samples were milled with potassium bromide $(\mathrm{KBr})(1 / 100)$ and pressed in a hydraulic press to small tablets. The FT-IR spectra were recorded on a Spectrum One FT-IR (Perkin Elmer) spectrometer equipped with a Multiscope (Perkin Elmer) microscopy. Each spectrum in a range of 4000 to $450 \mathrm{~cm}^{-1}$ was derived from 8 signal-averaged scans collected at a spectral resolution of 4 $\mathrm{cm}^{-1}$.

Liquid samples were analyzed placing a drop of the sample between plates of sodium chloride; analysis of data was carried in the range from 4000 to $600 \mathrm{~cm}^{-1}$. For each spectrum, 4 interferograms were averaged at a spectral resolution of $4 \mathrm{~cm}^{-1}$.

Skin samples were analyzed using $\mathrm{BaF}_{2}$ plates using Attenuated Total Reflectance FT-IR (ATR-FT$I R)$ tecnique. Each spectrum was derived from 128 signal-averaged scans collected at a spectral resolution of $4-\mathrm{cm}^{-1}$ The spectral window from 4000 to $700 \mathrm{~cm}^{-1}$ was chosen for identification.

\section{FT-IR imaging}

The spectra were collected using an Infrared Multichannel Viewer IMV-4000 (Jasco). Data were analysed using the software package Data Manager version 2 (Perkin Elmer). Each spectrum in a range of 4000 to $750 \mathrm{~cm}^{-1}$ was derived from 64 signal-averaged scans collected at a spectral resolution of $16-\mathrm{cm}^{-1}, 32-\mathrm{mm} / \mathrm{s}$ scan speed. 


\section{Statistical analysis of data}

Data analysis was carried out with the software package Microsoft Excel, version 2003.

Results are expressed as mean \pm standard deviation. One way analysis of variance (ANOVA) was used to substantiate statistical differences between groups, while Student's t-test was used for comparison between two samples. We used $\mathrm{P}<0.05$ as a minimal level of significance.

\section{Results and discussion}

In a previous work [15], liposomal vesicles prepared with penetration enhancers (PEVs) were characterized and their influence on MX accumulation was evaluated. All PEV formulations were able to incorporated a good amount of MX. Indeed, $\mathrm{E} \%$ ranged from $\approx 60 \%$ (PEVs with labrasol and transcutol) to $\approx 70 \%$ (PEVs with cineole). In particular, we demonstrated that the introduction of $\mathrm{PE}$ in the vesicular formulation led to deformable vesicles able to penetrate deeply in the skin with a formation of a drug depot.

In order to evaluate the effect of PEV incorporation on minoxidil skin (trans)dermal delivery, we carried out in vitro permeation study by applying MX loaded PEV formulations and conventional liposomes with the same lipid composition, but without PE, as control. All the experiments were carried out on the whole skin and in occlusive conditions. The choice of this model enabled us to perform measurements of the permeated as well as of the accumulated drug into the skin layers. To ascertain the role of the PEVs in the drug cutaneous delivery, permeation experiments were also carried out by pre-treating the skin with solutions of "free" PE and then applying MX loading liposomes. In table 1 the results of the experiments are reported.

The amount of MX detected in the skin layers, after PEVs treatment, was higher than that obtained placing the control formulation on the pre-treated skin specimens. Except for experiments with Transcutol $^{\circledR}$, the pre-treatment led to a reduction of the percentage of MX accumulated in the skin 
in comparison with results obtained when the chemical enhancer was a vesicle component (i.e. PEVs). By contrast, using Transcutol ${ }^{\circledR}$ as enhancer, the percentage of drug accumulated in the stratum corneum was approximately the double. Moreover, the same enhancer was responsible for the least transdermal permeation through the skin. The formation of the drug depot is due to swelling of the stratum corneum intercellular lipids caused by Transcutol ${ }^{\circledR}$. Inclusion of chemical enhancers in a liposomal formulation is capable of improving accumulation of minoxidil in the stratum corneum: that is due to the fact that these elastic vesicles do not travel beyond this skin layer and the permeation into the deep layers requires both drug release from the vesicles and its diffusion.

Then, FTIR spectroscopy was used to analyse the vesicular formulations and to study the diffusion and distribution of MX in the skin.

The first step of the study was to evaluate if the drug identification in the colloidal system was possible. MX, each liposome component and vesicular dispersion were analysed by FTIR.

A vesicular dispersion containing only soy lecithin and dicetylphosphate was also prepared and the FTIR spectrum was compared with those of free minoxidil and minoxidil containing vesicular systems. As shown in Fig 1, the MX stretchings corresponding to $\mathrm{NH}_{2}\left(3451,3411 \mathrm{~cm}^{-1}\right),=\mathrm{N}-\mathrm{H}$ $\left(3371 \mathrm{~cm}^{-1}\right), \mathrm{C}=\mathrm{C}$ and $\mathrm{C}=\mathrm{N}\left(1644,1611,1555 \mathrm{~cm}^{-1}\right)$, pyrimidine $\mathrm{N}$-oxide $\left(1234 \mathrm{~cm}^{-1}\right)$ and the system bands in the fingerprint region $\left(874,859,849,822\right.$ and at $\left.756 \mathrm{~cm}^{-1}\right)$ are clearly visible in the spectrum of the MX containing liposomes.

FTIR analysis were then carried out on skin specimens which had been pre-treated with the enhancers used in PEV formulations and then treated with the PEVs or the control for an $8 \mathrm{~h}$ in vitro permeation experiment. Before the analysis, the epidermis layer was compressed with an hydraulic press to obtain samples more homogeneous and with the same thickness, to avoid saturated signals, thus, allowing a comparative analysis of absorption band intensities. After the compression procedure, the skin samples showed different reactions to the hydraulic pressure: the pre-treated 
specimens tended to break, while those treated with PEVs were more elastic and underwent a deformation without any rupture.

To verify the MX penetration into the skin, we used the spectral window between 900 and $700 \mathrm{~cm}^{-1}$; in the other spectral regions, the SC absorption bands overlapped those of the vesicular components. FTIR spectra of the samples of PEVs treated skin showed well-defined absorption bands in the fingerprint region, a fact that may be attributed to the MX penetration into the skin. Although the enhancers analyzed separately showed absorption bands in that region, the signals, due to the low concentrations employed, are not visible in the skin, neither pretreated nor treated with PEVs (Fig.2, $3,4)$.

FTIR analysis confirmed the topical absorption of MX: the use of the PEVs led to a topical accumulation of the drug higher than that obtained with the control.

FTIR imaging spectroscopy has been shown to be a useful tool for studying molecule distribution in SC because it offers the possibility to obtain spatial information of the skin. FTIR images are formed on the basis of the contrast provided by the characteristic frequencies of a unique absorption band of the various components in the analyzed sample.

To investigate the distribution of MX loaded vesicles in the skin specimens, we carried out an FTIR imaging study. The skin samples were pressed to thin it to an appropriate thickness. FTIR images allowed us to verify the uniform thickness of the skin specimens. By recording images using a specific marker band both for liposomes and epidermis, it is possible to obtain maps whose superposition gives information about the vesicle penetration into the skin layers.

Fig. 5 shows the map obtained analyzing a skin sample treated with the vesicular dispersion containg Labrasol ${ }^{\circledR}$. Blue is used to report the absorption at $1735 \mathrm{~cm}^{-1}$ of the SL phosphate groups of MX liposomes (Fig.5a), while red represents the absorption at $1527 \mathrm{~cm}^{-1}$, associated to epidermis components (Fig.5b). The superposition of the two images (Fig. 5c), confirmed a distribution of MX loaded vesicles in all the skin surface, and showed both vesicles adsorption and absorption. 


\section{Conclusion}

In the present study, we investigated by FTIR analysis the ability of vesicles containing different penetration enhancers to increase MX accumulation in the upper skin layers. FTIR analysis showed the different drug behavior in the skin after treatment with PEVs or pretreatment with penetration enhancers and subsequent application of the MX liposomal dispersion (control).

Analytical results confirmed that the vesicle formulations with penetration enhancers promoted drug deposition in the skin compared to the control. FTIR imaging spectroscopy confirmed the vesicle distribution in the stratum corneum.

\section{Acknowledgments}

We thank Prof. Riccardo Stradi, University of Milan, Italy, for helpful discussions concerning the FTIR data.

The authors also thank Allevado (Allevatori Associati del Parteolla) Soc. Coop. A.R.L. for kindly supplying new born pig skin.

Declaration of interest: The authors declare that they do not have any direct financial relation with Gattefossè, (Saint Priest, France) and report no conflicts of interest. The authors alone are responsible for the contents and writing of the paper. 


\section{References}

[1] Tata S, Flynn GL, Weiner ND. Penetration of minoxidil from ethanol/propylene glycol solution: effect of application volume and occlusion. J Pharm Sci 1995; 84:688-691.

[2] Messenger A. Minoxidil: mechanism of action on hair growth. Brit J Dermatol 2004; 150:186-194.

[3] Pavithran K. Erythema multiforme following topical minoxidil. Indian J Dermatol Venereol Leprol 1993; 59:313-314.

[4] Sentjurc M, Vrhovnik K, Kristl J. Liposomes as a topical delivery system: the role of size on transport studied by EPR imaging method. J Control Release 1999; 59:87-97.

[5] Mezei M, Gulasekharam V. Liposomes: selective drug delivery system for the topical route of administration: Lotion dosage form. Life Sci 1980; 26:1473-1477.

[6] Mezei M, Gulasekharam V. Liposomes a selective drug delivery system for the topical route of administration: gel dosage form. J Pharm Pharmacol 1982; 34:473-474.

[7] Touitou E, Junginger HE, Weiner ND, Nagai T, Mezei M.. Liposomes as carrier for topical and transdermal delivery. J Phar Sci 1994; 83:1189-1203.

[8] Barry BW. Novel mechanism and devices to enable successful transdermal drug delivery. Eur J Pharm Sci 2001; 14:101-114. 
[9] Budai M, Szogyi M. Liposomes as drug carrier systems. Preparation, classification and therapeutic advantages of liposomes. Acta Pham Hung 2001; 71:114-118.

[10] Cevc G. Lipid vesicle and other colloids as drug carriers on the skin. Adv Drug Deliver Rev 2004; 56:675-711.

[11] Sinico C, Manconi M, Peppi M, Lai F, Valenti D, Fadda A.M. Liposomes as carriers for dermal delivery of tretinoin: in vitro evaluation of drug permeation and vesicle-skin interaction. J Control Release 2005; 103:123-136.

[12] Manconi M, Sinico C, Valenti D, Lai F, Fadda AM Niosomes as carriers for tretinoin: III. A study into the in vitro cutaneous delivery of vesicle-incorporated tretinoin. Int J Pharm 2006; 311:11-19.

[13] El Maghraby GM, Williams AC, Barry BW. Interactions of surfactants (edge activators) and skin penetration enhancers with liposomes. Int J Pharm 2004; 276:143-161.

[14] Mura S, Pirot F, Manconi M, Falson F, Fadda AM. Liposomes and niosomes as potential carriers for dermal delivery of minoxidil. J Drug Target 2007; 15:101-108.

[15] Mura S, Manconi M, Sinico C, Valenti D, Fadda AM. Penetration enhancers-containing vesicles (PEVs) as carriers for cutaneous delivery of minoxidil. Int J Pharm 2009; 380:7279. 
[16] Klimich HM, Chandra G. Use of Fourier transformed infrared spectroscopy with alternate total reflectance for in vivo quantification of polydimethylsiloxanes on human skin. J Soc Cosm Chem 1986; 37:73-87.

[17] Wartewig S, Neubert RHH. Pharmaceutical applications of Mid-IR and Raman spectroscopy. Adv Drug Deliv Rev 2005; 57:1144-1170.

[18] Harrison JE, Watkinson AC, Green DM, Hadgraft J, Brain K. The relative effect of Azone $^{\circledR}$ and Transcutol $^{\circledR}$ on permanent diffusivity and solubility in human stratum corneum. Pharm Res 1996; 13:542-546.

[19] Naik A, Pechtold LARM, Potts RO, Guy RH. Mechanism of oleic acid-induced skin penetration enhancement in vivo in humans. J Control Release 1995; 37:299-306.

[20] Potts RO, Guzek DB, Harris PR, McKie JE. A non-invasive, in vivo technique to quantitatively measure water concentration in the stratum corneum using attenuated total reflectance infrared spectroscopy. Arch Dermatol Res 1985; 277:489-495.

[21] Mak VHW, Potts RO, Guy RH. Percutaneous penetration enhancements in vivo measured by attenuated total reflectance infrared spectroscopy. Pharm Res 1990; 7:835-841.

[22] Reinl HM, Hartinger A, Dettmar P, Bayrel TM. Time resolved infrared ATR measurement of liposome transport kinetics in human keratinocyte cultures and skin reveals a drastic dependence on liposome size and phase state. J Invest Dermatol 1995; 105:291-295. 
[23] Kezic S. Methods for measuring in-vivo percutaneous absorption in humans. Hum Exp Tossicol 2008; 27:289-295.

[24] Laugel C, Rafidison P, Potard G, Aguadisch L, Baillet A. Modulated release of triterpenic compounds from $\mathrm{O} / \mathrm{W} / \mathrm{O}$ multiple emulsion formulated with dimethicones: infrared spectrofhotometric and differencial calorimetric approaches. J Control Release 2000; 63:717.

[25] Laugel C, Yagoubi N, Baillet A. ATR-FTIR spectroscopy: a chemiometric approach for studying the lipid organization of stratum corneum. Chem Phys Lipids 2005; 135:55-68. 


\section{CAPTIONS OF FIGURES}

Fig.1: FTIR spectra of vesicular dispersion (a),minoxidil (b) and vesicular dispersion containing minoxidil (c) in the range between $4000-2600 \mathrm{~cm}^{-1}$ (A) and $2000-700 \mathrm{~cm}^{-1}$ (B)

Fig. 2: FTIR spectra of skin sample (a), skin pretreated with Labrasol and than with liposome (b), skin treated with minoxidil containing PEV (c) in the range between $4000-2600 \mathrm{~cm}^{-1}$ (A) and 2000$700 \mathrm{~cm}^{-1}(\mathrm{~B})$

Fig. 3: FTIR spectra of skin sample (a), skin pretreated with Cineole and than with liposome (b), skin treated with minoxidil containing PEV (c) in the range between 4000-2600 $\mathrm{cm}^{-1}$ (A) and 2000$700 \mathrm{~cm}^{-1}(\mathrm{~B})$

Fig. 4: FTIR spectra of skin sample (a), skin pretreated with Transcutol and than with liposome (b), skin treated with minoxidil containing PEV (c) in the range between 4000-2600 $\mathrm{cm}^{-1}$ (A) and 2000$700 \mathrm{~cm}^{-1}(\mathrm{~B})$

Fig 5: Absorption of the phosphate groups of soy lecithin at $1735 \mathrm{~cm}^{-1}$ (a); represent the absorption at $1527 \mathrm{~cm}^{-1}$, associated to epidermis components (b); superposition of a and b (c) 
Table 1. Results of in vitro permeation study after treatment of pig skin with PEV suspensions or after pre-treatment of pigskin with penetration enhancer solutions followed by treatment with conventional MX loaded liposomes (control): percentage of MX accumulated into and delivered through the skin at the end of the experiments. Each data is the mean \pm standard deviation of at least six experimental determinations.

\begin{tabular}{lcccc}
\hline & \multicolumn{3}{c}{ Dose minoxidil (\%) } \\
\cline { 2 - 5 } Formulation & SC & Epidermis & Dermis & Receptor fluid \\
\hline Control & $4.02 \pm 0.15$ & $2.33 \pm 0.04$ & $0.08 \pm 0.01$ & n.d. \\
PEV (labrasol) & $11.07 \pm 0.77$ & $8.95 \pm 1.07$ & $0.02 \pm 0.01$ & n.d. \\
PEV (transcutol) & $4.99 \pm 0.42^{*}$ & $2.82 \pm 0.38^{*}$ & $0.06 \pm 0.01$ & n.d. \\
PEV (cineole) & $12.85 \pm 1.07$ & $4.04 \pm 0.09$ & $0.09 \pm 0.02$ & n.d. \\
Labrasol + control & $6.19 \pm 1.46$ & $2.17 \pm 0.33$ & $0.04 \pm 0.004$ & $0.41 \pm 0.18$ \\
Transcutol + control & $8.86 \pm 0.71$ & $2.88 \pm 0.28$ & $0.17 \pm 0.002$ & $0.28 \pm 0.17$ \\
Cineole + control & $7.01 \pm 2.13$ & $3.18 \pm 0.65$ & $0.004 \pm 0.001$ & $4.71 \pm 1.09$ \\
\hline *p< 0.05 in comparison with control $(\mathrm{n}=9)$ & & & \\
n.d. not determined & & & &
\end{tabular}




\section{Figure 1}

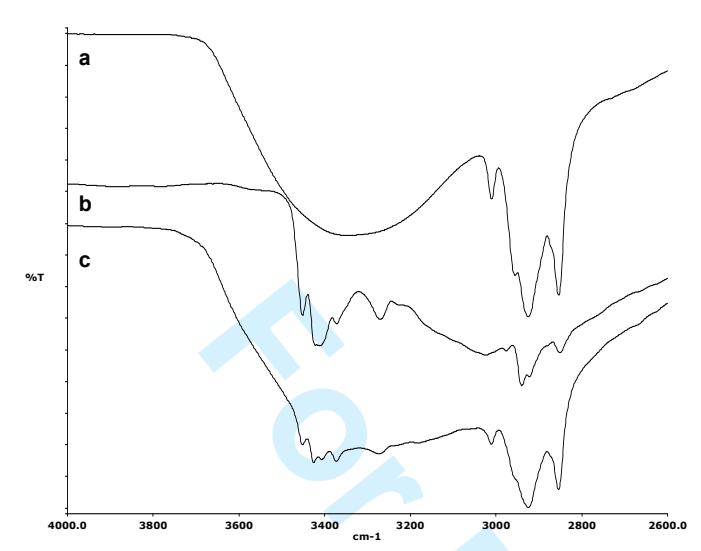

A

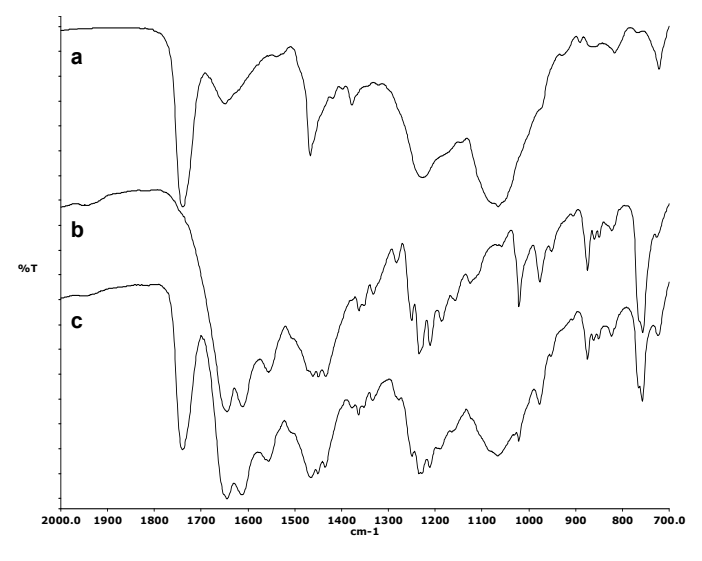

B 


\section{Figure 2}
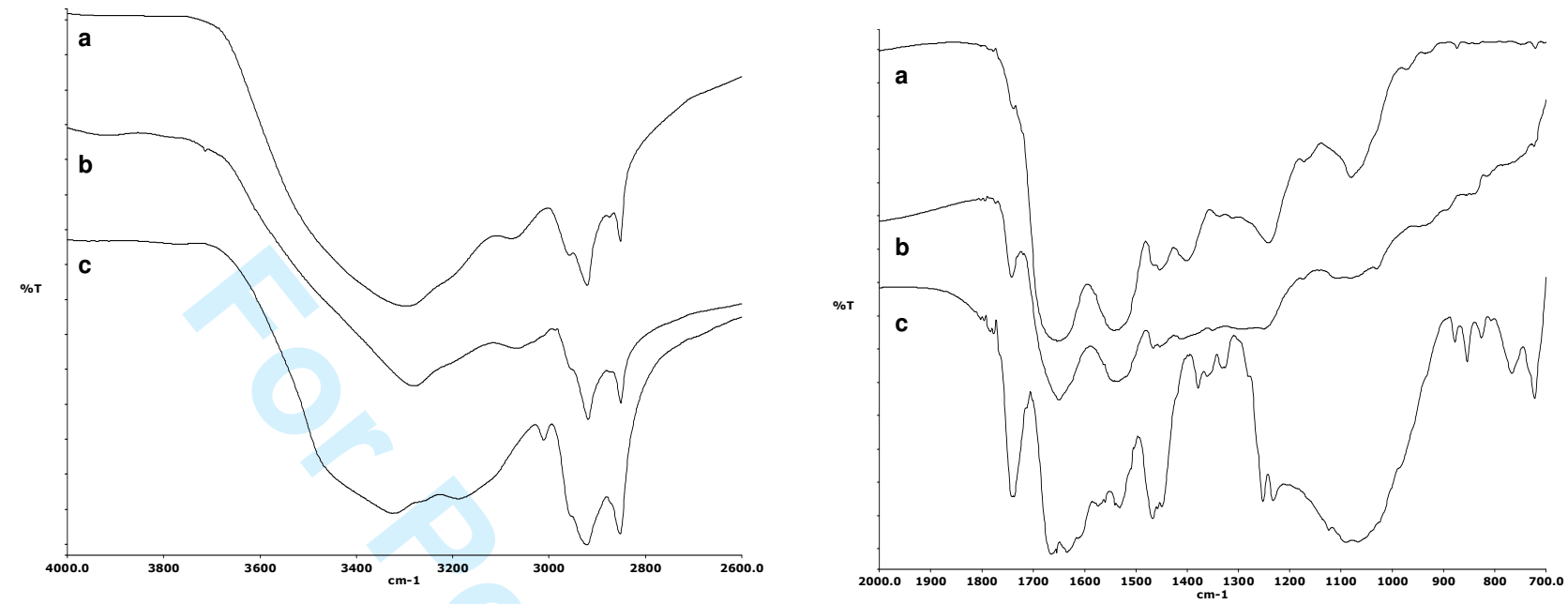

A

B 


\section{Figure 3}

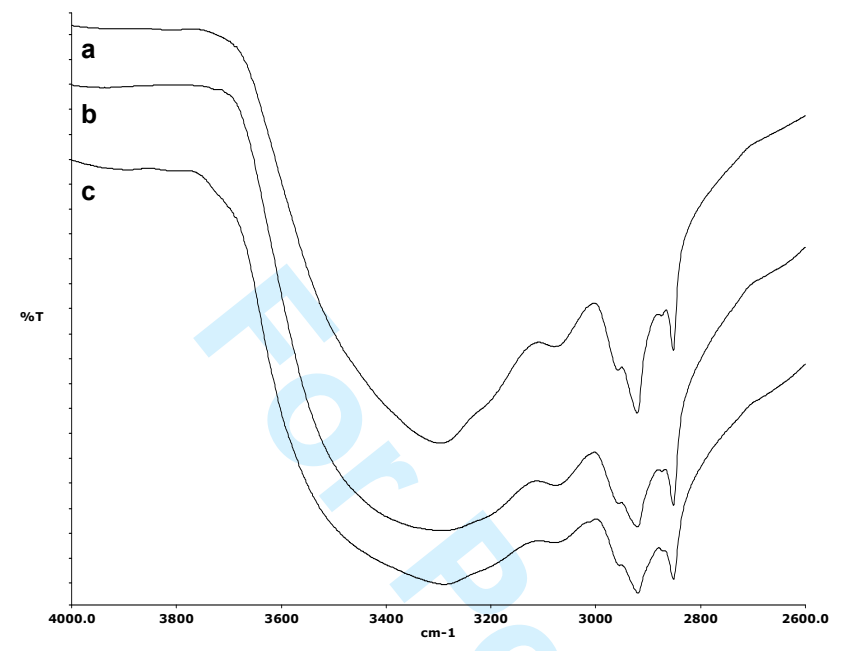

A

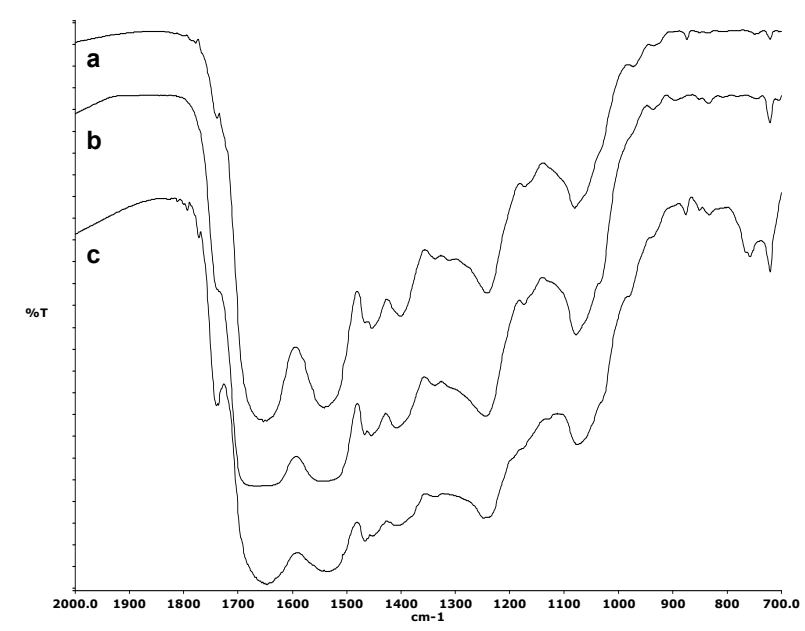

B 


\section{Figure 4}

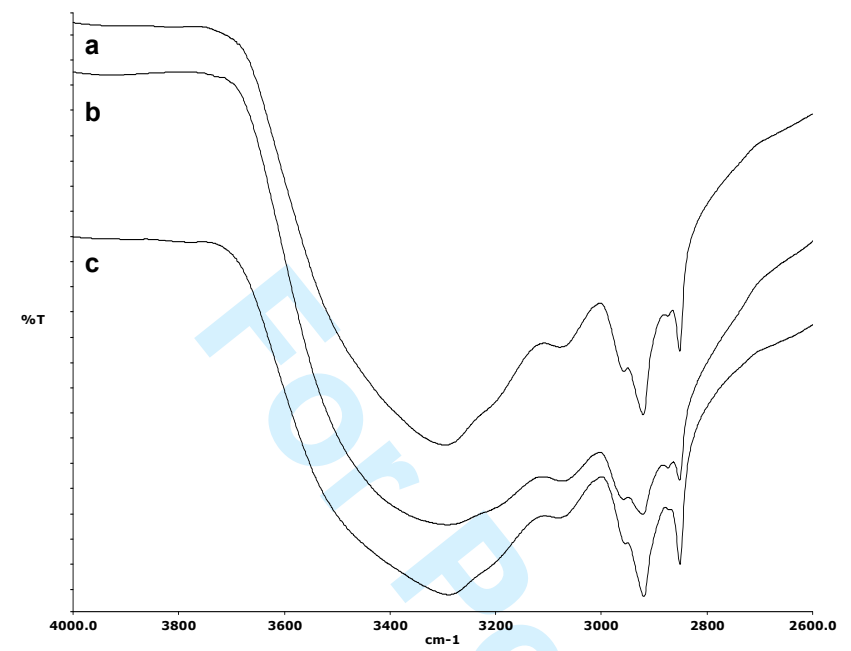

A

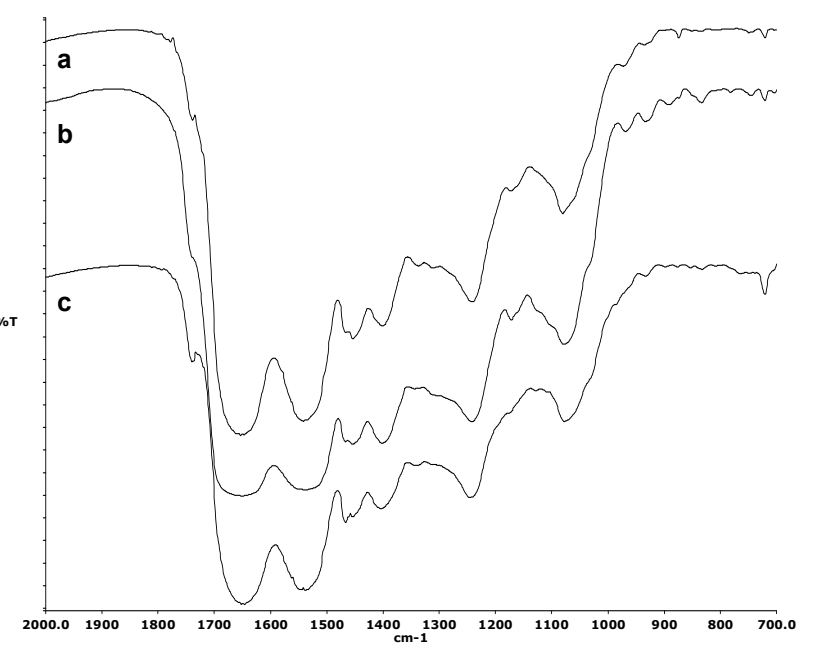

B

URL: http://mc.manuscriptcentral.com/lpdt 
1

2

3

4

5

6

7

8

9

10

11

12

13

14

15

16

17

18

19

20

21

22

23

24

25

26

27

28

29

30

31

32

33

34

35

36

37

38

39

40

41

42

43

44

45

46

47

48

49

50

51

52

53

54

55

56

57

58

59

60

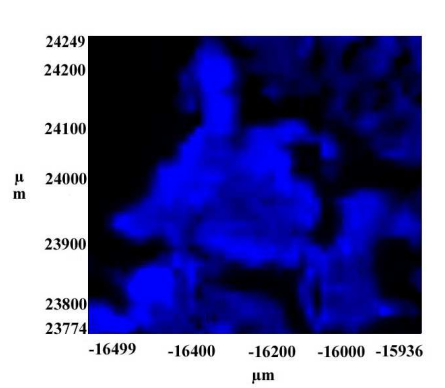

(a)

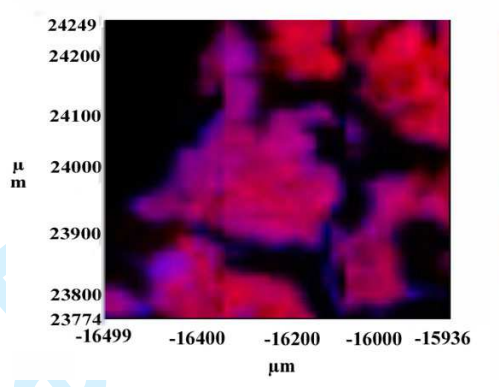

(c)

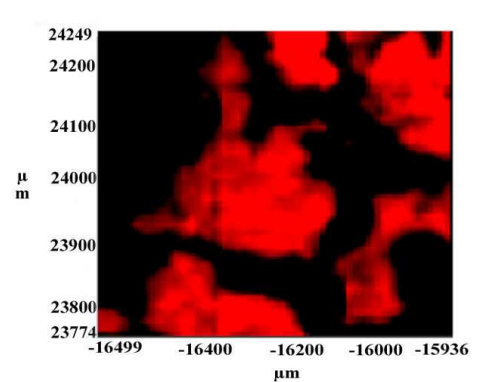

(b)

Figure 5
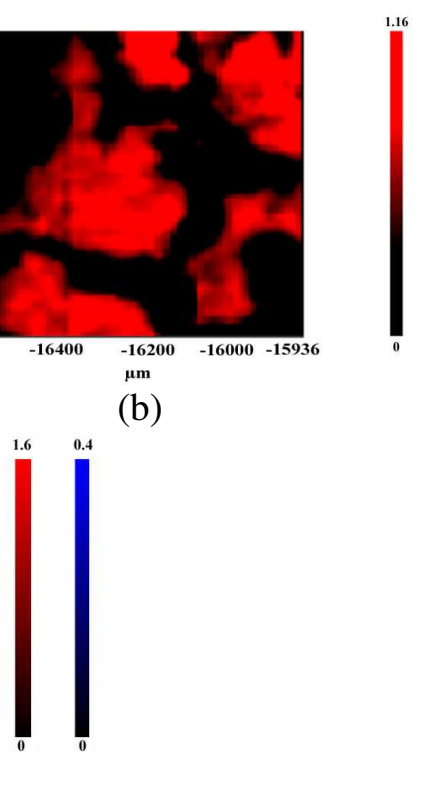